\title{
Metastable and stable equilibrium states of stellar electron-nuclear plasmas
}

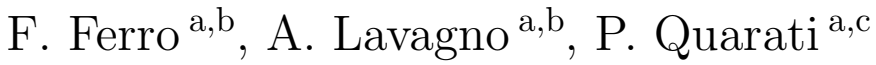 \\ ${ }^{a}$ Dipartimento di Fisica, Politecnico di Torino, I-10129 Torino, Italy \\ ${ }^{\mathrm{b}}$ INFN - Sezione di Torino, I-10125 Torino, Italy \\ ${ }^{\mathrm{c}}$ INFN - Sezione di Cagliari, I-09042 Monserrato, Italy
}

\begin{abstract}
By minimizing free energy density, we show that the stellar core of a hydrogen burning star is not in a global thermodynamical equilibrium unless density, temperature, mass and composition assume given values. The core (as the solar interior) may be viewed more appropriately as a metastable state with very long lifetime. Slightly non-extensive distribution function could be the natural distribution for a weakly non-ideal plasma like a stellar core and represents a more appropriate approximation to this system than a Maxwellian distribution, without affecting bulk properties of stars.
\end{abstract}

Key words: Non-extensive distribution function; metastability conditions; weakly non-ideal plasma.

PACS: 05.90.+m; 95.30.Qd.

\section{Introduction}

Landau and Lifšits [1] distinguish between two different equilibrium states called metastable and stable states. A system in a metastable state cannot return to its initial state after a sufficiently large deviation from it and could pass to another (meta)stable state. Although metastable states are stable within certain limits, after a time that could be very long, the system will pass to another state. A system removed from the stable state with maximum entropy will sooner or later return to it.

Sewell [2] shows that the equilibrium states are globally thermodynamically stable if they minimize the free energy density of the system, satisfy KMS (Kubo-Martin-Schwinger) fluctuation-dissipation conditions and are of Gibbsian type. Metastable states (ideal with infinite lifetime or normal with finite 
long lifetime) have a minimum of free energy density only in some reduced space state and these states are always supported only by models with manybody, long-range interactions among the particles (as, for instance, gravitational, Coulomb, spin-glass interactions). Considering infinite systems, Sewell minimizes density of free energy to avoid divergences. The minimization of this quantity allows us to analyze local, finite subsystems of the entire system (like, for instance, as small as the Debye-Hückel plasma sphere).

Recently, it has been shown that metastable states can naturally be described by distributions accounting for temperature fluctuations [3,4] or by quasiparticle models [5], and belonging to the generalized statistical mechanics that now is usually called non-extensive thermostatistics [6].

In this work we are interested in non-relativistic, hot, dense electron-nuclear plasmas (like the core of stars or the solar interior) and discuss the conditions required for their equilibrium or their metaequilibrium (metastability), following the approach outlined by Landau and Lifšits [7] and recently applied by Vasiliev [8], but minimizing free energy density and total energy density.

It is commonly accepted that stars like the Sun have a core, i.e. an electronnuclear plasma, in equilibrium conditions, that can be described by an electron and an ion velocity distribution which are Maxwellian (local thermodynamical equilibrium).

Main sequence stars produce a luminosity equal to the heat production rate and reach a stationary regime. Nuclear fusion reactions produce the heat and do not sensibly perturb the state of the Sun. This stationary state that is usually called, in astrophysics, equilibrium state, has a long life-time, until the nuclear fuel is completely burned out. Stars are not in thermal equilibrium at present because they radiate and produce heat in the core with a temperature decreasing from core to surface. Thermal exchanges take a very long time, whereas gravitational equilibrium is rapidly reached. Nuclear reactions are so slow that in the time interval between one fusion reaction and another the system returns to the Maxwellian equilibrium due to many binary Coulomb collisions among the ions [9].

We want to point out that the return to the initial state after a fusion reaction is also possible if the distribution function describing the initial state is a stationary (stable or metastable) one, analytically expressed by a deformed Maxwellian (e.g. a Druyvenstein distribution) or a non-extensive distribution. What counts to decide the formal expression of the distribution is the type of collisions in which the ions are involved and the dependence on momentum of the elastic collisional cross sections (Coulomb, screened Coulomb, enforced Coulomb, among others), or the complete absence of ion-ion correlation and interaction [10]. Therefore, this argument based on the slowness of fusion 
reactions is not useful to decide whether the system (the electron-nuclear core) is really in an equilibrium state (globally thermodynamically stable) or is close to it, or rather it is a metastable state, and to decide what distribution is the best approximation.

In this work, we deduce the values of temperature, density and core radius of stellar interiors having a given composition at which they are in equilibrium (or in metaequilibrium state), supposing stellar cores made of ideal ionized gas plus corrections due to particles identity and internuclear interactions, as indicated in Refs. [7,8].

We find that globally thermodynamically stable equilibrium is reached in stars of the same size and composition of the Sun, at the core thermal energy of $\mathrm{k}_{\mathrm{B}} T \approx 5 \mathrm{keV}$ (more than three times higher than the actual temperature of the solar interior), and at an electron density of $n \approx 0.027 \cdot 10^{-12} \mathrm{fm}^{-3}$ (about half the actual value in the solar core, that must have a volume of the central core of radius $R \approx 0.2 R_{\odot}$ ).

Therefore, Maxwellian distributions are of course very good approximations (zero approximations), considering the high precision reached by SSM (Standard Solar Model) in describing solar neutrinos production. However a better approximation should be the distribution of a metastable state, possibly given in an effort to accommodate equilibrium and metaequilibrium states, as recently developed in non-extensive thermostatistics, in spite of criticism devoted to its use in this field $[11,12]$.

As already shown in the recent past, very small deviations from Maxwellian momentum distribution do not modify the properties of stellar core, are in agreement with the helioseismology constraints, but may affect the evaluation of the nuclear fusion rates that may be enhanced or depleted, depending on superdiffusion or subdiffusion property of the particles [13]. Let us mention that deformed distributions have been recently used also in the interpretation of many heavy ions processes in thermalization and equilibration of quarkgluon plasma [14]; a study of big bang nucleosynthesis of lithium and of a possible solution of the problem of lithium abundance in astrophysical systems have been undertaken by us and is in progress, among other applications [6].

\section{Metastable states}

Effort to accommodate a fundamental characterization of either equilibrium or metastable states in terms of certain stability conditions was undertaken since long time. The equilibrium states are globally thermodynamically stable, and are those that minimize the free energy density of the system and satisfy 
KMS conditions. Grades of metastability correspond to different local stability properties.

Metastable states (ideal) do not minimize free energy density of the entire system, but minimize the restriction of free energy density to some reduced state space. They are long-lived, supported by long-range, many-body forces. They are locally thermodynamically stable if the free energy of the system cannot be lowered by modifications of the state that is confined to bounded regions of space [2].

The metastability of a state depends on the thermal perturbations to which the system is subjected. A state may have very long lifetime in presence of certain thermal perturbations and may be short-lived when subjected to others. Of course, in presence of perturbations or thermal fluctuations, the distribution function of the system differs more or less from the Maxwellian distribution.

By using well known results of Landau and Lifšits [7], and following the approach of Vasiliev [8], we examine the conditions for equilibrium by minimizing the free energy density. The free energy of an ideal electron gas is

$$
F_{\text {ideal }}=-\mathrm{k}_{\mathrm{B}} T N-\mathrm{k}_{\mathrm{B}} T N \ln \left\{\frac{1}{n}\left[\frac{m \mathrm{c}^{2} \mathrm{k}_{\mathrm{B}} T}{2 \pi(\hbar \mathrm{c})^{2}}\right]^{3 / 2}\right\},
$$

where $N$ and $m$ are, respectively, the number and the mass of electrons, $n$ is the electron density, $\mathrm{k}_{\mathrm{B}} T$ is the thermal energy of the plasma.

Let us consider now a hot dense electron-nuclear plasma: the free energy should be written, starting from Eq. (1), in the following form

$$
F=F_{\text {ideal }}+F_{\text {quantum }}+F_{\text {correlations }}
$$

where $F_{\text {quantum }}$ and $F_{\text {correlations }}$ account for the main corrections that arise in real gases, namely the quantum exclusion principle between fermions and correlation effects between charged particles.

The first correction, $F_{\text {quantum }}$, can be written as

$$
F_{\text {quantum }}=+N E_{1} n
$$

with

$$
E_{1}=\left(\frac{\pi^{3 / 2} \mathrm{a}_{0}^{3 / 2} \mathrm{e}^{3}}{4}\right) \frac{1}{\left(\mathrm{k}_{\mathrm{B}} T\right)^{1 / 2}}=\frac{e_{1}}{\left(\mathrm{k}_{\mathrm{B}} T\right)^{1 / 2}},
$$


where $\mathrm{a}_{0} \simeq 0.53 \cdot 10^{5} \mathrm{fm}$ is the Bohr radius, and $e_{1} \simeq 2.68 \cdot 10^{7} \mathrm{MeV}^{3 / 2} \mathrm{fm}^{3}$.

The second correction is

$$
F_{\text {correlation }}=-N E_{2} n^{1 / 2},
$$

with

$$
E_{2}=\left(\frac{2 \pi^{1 / 2} \mathrm{e}^{3}}{3}\right) \frac{(\bar{Z}+1)^{3 / 2}}{\left(\mathrm{k}_{\mathrm{B}} T\right)^{1 / 2}}=e_{2} \frac{(\bar{Z}+1)^{3 / 2}}{\left(\mathrm{k}_{\mathrm{B}} T\right)^{1 / 2}},
$$

where $\bar{Z}$ is the average chemical composition and $e_{2} \simeq 2.04 \mathrm{MeV}^{3 / 2} \mathrm{fm}^{3}$.

If we define

$$
a=\left[\frac{m \mathrm{c}^{2} \mathrm{k}_{\mathrm{B}} T}{2 \pi(\hbar \mathrm{c})^{2}}\right]^{3 / 2}=a_{1}\left(\mathrm{k}_{\mathrm{B}} T\right)^{3 / 2},
$$

where $a_{1} \simeq 0.28 \cdot 10^{-8} \mathrm{MeV}^{-3 / 2} \mathrm{fm}^{-3}$, the free energy density, starting from Eqs.(2), (3) and (4), is

$$
\frac{F}{V}=-\mathrm{k}_{\mathrm{B}} T n-\mathrm{k}_{\mathrm{B}} T n \ln \left(\frac{a}{n}\right)+E_{1} n^{2}-E_{2} n^{3 / 2},
$$

$V$ being the volume of the plasma.

Now, we should calculate the value $n_{*}$ of particle density that minimizes the $F / V$ free energy density: thus, we can write the extremal condition

$$
\left.\frac{\partial(F / V)}{\partial n}\right|_{T, N}=0
$$

from which we obtain the non-linear equation

$$
\ln \left[\frac{n}{a_{1}\left(\mathrm{k}_{\mathrm{B}} T\right)^{3 / 2}}\right]+2 \frac{e_{1}}{\left(\mathrm{k}_{\mathrm{B}} T\right)^{3 / 2}} n-\frac{3}{2} e_{2} \frac{(\bar{Z}+1)^{3 / 2}}{\left(\mathrm{k}_{\mathrm{B}} T\right)^{3 / 2}} n^{1 / 2}=0,
$$

that links together $T, n$ and the chemical composition $\bar{Z}$.

It can be shown that condition (5) is not only necessary, but also sufficient to assure that the root of Eq.(6) is the actual minimum of free energy density, 
provided that the inequality

$$
\frac{(\bar{Z}+1)^{3}}{\left(\mathrm{k}_{\mathrm{B}} T\right)^{3 / 2}}<9.17 \cdot 10^{7} \mathrm{MeV}^{-3 / 2}
$$

is satisfied. Astrophysical plasmas of stellar cores usually have $\mathrm{k}_{\mathrm{B}} T \sim 1 \mathrm{keV}$ and $\bar{Z}$ of order unity: then the previous inequality holds for these systems.

Looking for a second relation linking $n$ and $T$, we can take the total energy of non-relativistic electron-nuclear plasma as

$$
E_{\text {total }}=U+E_{\text {kinetic }}+\frac{3}{2} N E_{1} n-\frac{3}{2} N E_{2} n^{1 / 2}+\frac{\pi^{2}}{15}\left(\frac{\mathrm{k}_{\mathrm{B}} T}{\hbar \mathrm{c}}\right)^{3} V \mathrm{k}_{\mathrm{B}} T,
$$

where the last term represents the black-body radiation contribution.

According to virial theorem [15], the potential energy $U$ of particles with Coulomb interaction is equal to their double kinetic energy with opposite sign, namely

$$
U=-2 \frac{3}{2} N \mathrm{k}_{\mathrm{B}} T=-3 N \mathrm{k}_{\mathrm{B}} T
$$

According to Eqs. (7) and (8), we obtain the total energy density

$$
\frac{E_{\text {total }}}{V}=-\frac{3}{2} \mathrm{k}_{\mathrm{B}} T n+\frac{3}{2} \frac{e_{1}}{\left(\mathrm{k}_{\mathrm{B}} T\right)^{1 / 2}} n^{2}-\frac{3}{2} \frac{e_{2}(\bar{Z}+1)^{3 / 2}}{\left(\mathrm{k}_{\mathrm{B}} T\right)^{1 / 2}} n^{3 / 2}+\frac{\pi^{2}}{15} \frac{\left(\mathrm{k}_{\mathrm{B}} T\right)^{4}}{(\hbar \mathrm{c})^{3}}
$$

whose minimum condition,

$$
\left.\frac{\partial\left(E_{\text {total }} / V\right)}{\partial\left(\mathrm{k}_{\mathrm{B}} T\right)}\right|_{N, V}=0
$$

can now be written as

$$
-\frac{3}{2} n-\frac{3}{4} \frac{e_{1}}{\left(\mathrm{k}_{\mathrm{B}} T\right)^{3 / 2}} n^{2}+\frac{3}{4} \frac{e_{2}(\bar{Z}+1)^{3 / 2}}{\left(\mathrm{k}_{\mathrm{B}} T\right)^{3 / 2}} n^{3 / 2}+\frac{4 \pi^{2}}{15}\left(\frac{\mathrm{k}_{\mathrm{B}} T}{\hbar \mathrm{c}}\right)^{3}=0 .
$$

Eq. (9) is the second equation that links $T, n$ and $\bar{Z}$ together.

Considering that, in a Sun-like star, $n \sim 10^{-14} \mathrm{fm}^{-3}, \mathrm{k}_{\mathrm{B}} T \sim 10^{-3} \mathrm{MeV}$ and $\bar{Z} \sim 1.25$, we can neglect the terms with $e_{1}$ and $e_{2}$ in Eq. (9), thus obtaining

$$
\mathrm{k}_{\mathrm{B}} T=\left[\frac{3}{2} n \frac{15}{4 \pi^{2}}(\hbar \mathrm{c})^{3}\right]^{1 / 3}=b^{1 / 3} n^{1 / 3}
$$


where $b \simeq 4.56 \cdot 10^{6} \mathrm{MeV}^{3} \mathrm{fm}^{3}$.

Therefore, $\mathrm{k}_{\mathrm{B}} T$ and $n$ must satisfy both Eq. (6) and Eq. (10), given a fixed chemical composition, $\bar{Z}$. If we choose $\bar{Z}=1.25$, i.e. the Sun composition, we find out from the previous discussion that the plasma is globally thermodynamically stable if and only if its electron density $n_{*}$ and its thermal energy $\mathrm{k}_{\mathrm{B}} T_{*}$ are, respectively,

$$
n_{*} \simeq 2.74 \cdot 10^{-14} \mathrm{fm}^{-3} \text { and } \mathrm{k}_{\mathrm{B}} T_{*} \simeq 5 \cdot 10^{-3} \mathrm{MeV}
$$

Let us now consider a star characterized by the values of $n_{*}$ and $\mathrm{k}_{\mathrm{B}} T_{*}$ given above in Eq. (11); we want to determine if its mass, $M_{*}$, could satisfy the condition $M_{*} \approx M_{\odot}$, where $M_{\odot} \simeq 1.99 \cdot 10^{33} \mathrm{~g}$ is the solar mass. We suppose the star in hydrostatic equilibrium, namely the gravitational pressure, which tends to compress the star, is exactly counterbalanced by the internal pressure of the hot gas and by the pressure of radiation. Therefore, the static-equilibrium equation is

$$
\frac{G M^{2}}{6 R V}=\mathrm{k}_{\mathrm{B}} T n+\frac{\pi^{2}}{15} \frac{\left(\mathrm{k}_{\mathrm{B}} T\right)^{4}}{(\hbar \mathrm{c})^{3}},
$$

with $V \propto R^{3}$.

If we impose that $n \equiv n_{*}, \mathrm{k}_{\mathrm{B}} T \equiv \mathrm{k}_{\mathrm{B}} T_{*}$ and $M \equiv M_{*} \equiv M_{\odot}$, we obtain the following relation between the radius of a stellar core in equilibrium conditions, $R_{*}$, and the actual solar radius, $R_{\odot}$,

$$
R_{*} \simeq 0.21 R_{\odot}
$$

By comparing the quantities $n, T$ and $M$, we have seen, through Eq. (12), that the core of a star like the Sun is not in an equilibrium state globally thermodynamically stable, although the equilibrium Maxwellian distribution, used for solar core description, is a good approximation. The core can be better described as a metaequilibrium or metastable state. This state may be due to temperature fluctuations [4] or quasi-particle behavior of the ions of plasma [5].

\section{The number of particles inside the Debye-Hückel sphere}

Before reporting on the application to main sequence stars (with masses close to the solar mass) of free energy density minimization, we wish to recall the 
fully analytical descriptions of stellar models and stellar stability by Clayton [16], Nauenberg and Weisskopf [17], and Balian and Blaizot [18].

As reported in these papers, the equilibrium properties are obtained by hydrostatic equations with the addition of local thermodynamical equations for matter and radiation, based on Boltzmann-Gibbs entropy, with the consequence that stars evolve along a sequence of states in a complete hydrostatic and thermal equilibrium. However, as we shall verify, in these states physical quantities like density, temperature and core radius of stars with the composition and size of the Sun are not those required to achieve a global free energy density minimum, that, rather, is a local minimum with a very long lifetime, because of many-body interactions. Following Sewell, this state is a metastable state which (as shown for instance by Sakagami and Taruya [19]) can more conveniently be described by a non-extensive distribution function.

The star interior is a hot dense electron-nuclear plasma at high pressure and temperature. Density and temperature are growing toward the center, because we require that the system be stable in the gravitational field. At zero approximation, plasma core can be considered as a Boltzmann ideal gas, with energy $3 N k_{\mathrm{B}} T / 2$. SSM, based on ideal gas approximation of the core [20,21], correctly evaluates the measured solar neutrino fluxes. SSM cannot account for the existence of density perturbations since it is based on hydrostatic evolution equation.

In the hydrodynamical approximation, density perturbations can be induced by corresponding temperature fluctuations due to convection of matter between layers with different local temperatures.

Greater is the quantity $\left(\mathrm{k}_{\mathrm{B}} T\right)^{3 / 2} n^{-1 / 2}$ (where $n$ is, as usual, the number density), better approximation of the stellar core is the equilibrium Maxwellian distribution. In fact, this quantity is related to the square number $N_{\mathrm{D}}$ of particle inside the Debye-Hückel sphere, which must be sufficiently large in order to apply Boltzmann-Gibbs statistics.

If we define the volume of the Debye-Hückel sphere $V_{\mathrm{D}}$,

$$
V_{\mathrm{D}}=\frac{4}{3} \pi R_{\mathrm{D}}^{3}=\frac{4}{3} \pi\left(\frac{\mathrm{k}_{\mathrm{B}} T}{4 \pi \mathrm{e}^{2} \sum_{i} n_{i} Z_{i}^{2}}\right)^{3 / 2}
$$

and the number of particles $N_{\mathrm{D}}$ inside the Debye-Hückel sphere,

$$
N_{\mathrm{D}}=n V_{\mathrm{D}} \propto \frac{\left(\mathrm{k}_{\mathrm{B}} T\right)^{3 / 2}}{n^{1 / 2}}
$$

and assuming that $V_{\mathrm{D}}$ contains only protons, helium nuclei and electrons, for 
a star with composition similar to that of the solar core, we obtain $N_{\mathrm{D}} \approx 4$. This is a very small number to apply, without criticism, the Boltzmann-Gibbs statistics, because the fluctuations of that number are great. Furthermore, the value of $N_{\mathrm{D}}$ can increase if $\mathrm{k}_{\mathrm{B}} T$ increases and/or the density $n$ decreases.

Going on with time (few billion of years) the core of stars evolves toward states that are closer to equilibrium, but they deviate from this behavior when the helium burning becomes the most important fusion process. At $t \simeq 8 \cdot 10^{9} \mathrm{y}$ we have an electron-nuclear (hydrogen) plasma with a density lower than the actual density and a temperature much higher [22]; therefore this state (pre-helium burning state) is closer to global equilibrium and the Maxwellian distribution describing this state is a better approximation than at an earlier time because $\left(\mathrm{k}_{\mathrm{B}} T\right)^{3 / 2} n^{-1 / 2}$ is greater.

A state with a great value of $\left(\mathrm{k}_{\mathrm{B}} T\right)^{3 / 2} n^{-1 / 2}$ is closer to equilibrium than a state with a lower value. Let us also note that, although $R_{\mathrm{D}} \propto\left(\mathrm{k}_{\mathrm{B}} T / n\right)^{1 / 2}$ is constant along the star profile, $\left(\mathrm{k}_{\mathrm{B}} T\right)^{3 / 2} n^{-1 / 2}$ is not.

Of course, as $N_{D}$ increases, the weak electron screening correction

$$
f^{\mathrm{WES}} \approx \exp \left(\frac{1}{4 \pi} \frac{Z_{1} Z_{2}}{3 N_{\mathrm{D}}}\right)
$$

goes to one.

In non-extensive thermostatistics the free energy of an ideal gas (without considering corrections at the moment) is given by [23]

$$
F_{q}=-\mathrm{k}_{\mathrm{B}} T \ln _{q}\left[\Lambda d(q) V^{N_{\mathrm{D}}}\left(\mathrm{k}_{\mathrm{B}} T\right)^{3 / 2 N_{\mathrm{D}}}\right]
$$

where

$$
\Lambda=\frac{1}{N_{\mathrm{D}} !}\left(\frac{m}{2 \pi \hbar^{2}}\right)^{3 N_{\mathrm{D}} / 2},
$$

$\ln _{q} x=\left(x^{1-q}-1\right) /(1-q)$ is the $q$-logarithm and $d(q)$ is a suitable function of the entropic parameter. Let us apply the above expression of $F_{q}$ to the DebyeHückel sphere. When $q \neq 1$, in order to have a minimum of $F_{q}$, one needs a large value of $R_{D}$ that means a large value of $\left(\mathrm{k}_{\mathrm{B}} T\right)^{3 / 2} n^{-1 / 2}$. Of course, as $q \rightarrow 1$ the free energy reaches the minimum for fixed $T$ and $n$.

The entropy of the subsystem can be deduced from the relation

$$
F=T S-E_{\text {tot }}
$$


the expression of $F$ being well-known.

Neglecting two terms in comparison to others, we can write

$$
\frac{S}{\mathrm{k}_{\mathrm{B}}} \simeq \frac{5}{2} N_{\mathrm{D}}\left[+1+n \frac{e_{1}}{\left(\mathrm{k}_{\mathrm{B}} T\right)^{3 / 2}}-n^{1 / 2} e_{2} \frac{(\bar{Z}+1)^{3 / 2}}{\left(\mathrm{k}_{\mathrm{B}} T\right)^{3 / 2}}\right] .
$$

We know that the non-extensive Tsallis entropy can be written, for a system with a small $N_{\mathrm{D}}$, as $[24]$

$$
\frac{S_{q}}{\mathrm{k}_{\mathrm{B}}}=\frac{5}{2} N_{\mathrm{D}}\left[+1+\frac{1-q}{2} \frac{5}{2} N_{\mathrm{D}}\right]
$$

Equalling the two relations in Eqs. (13) and (14) and substituting $n_{*}$ and $T_{*}$ with $N_{\mathrm{D}} \approx 4$, we obtain that in the Debye-Hückel sphere the $q$ parameter must assume the value of $\approx 1.001$, in order to achieve equilibrium. The correction of one thousand, compared to unity, seems very negligible. However it can be not at all without importance in the calculation of the fusion rates.

\section{Distribution function of metastable states}

Non-equilibrium systems with metastable states can be composed of regions that show fluctuations in space and time of an intensive quantity like the inverse temperature $\beta[4]$.

Non-equilibrium metastable states of a macroscopic system are made of cells that are temporarily in local equilibrium. Each small region has a different $\beta$, varying in accordance with the probability distribution function $f(\beta)$. Therefore, stationary probability density of a non-equilibrium system are given by

$$
P(\varepsilon)=\int_{0}^{+\infty} \mathrm{d} \beta f(\beta) \mathrm{e}^{-\beta \varepsilon}
$$

The function $f(\beta)$ can assume several expressions. However, in case of small deviations from Maxwellian distribution, the $P(\varepsilon)$ function can always be written as $[4]$

$$
P(\varepsilon)=\mathrm{e}^{-\beta_{0} \varepsilon} \mathrm{e}^{-\delta\left(\beta_{0} \varepsilon\right)^{2}}=\mathrm{e}^{-\beta_{0} \varepsilon}\left[1+\frac{1}{2}(q-1) \beta_{0}^{2} \varepsilon^{2}+\ldots\right]
$$

where $\delta=(1-q) / 2, q$ is the Tsallis non-extensive parameter, and $\beta_{0}$ is the 
average value of the quantity $\beta$. In this case, if the distribution $f(\beta)$ has a standard deviation $\sigma$, we obtain the useful relation

$$
\sqrt{q-1}=\frac{\sigma}{\beta_{0}}
$$

$P(\varepsilon)$ is proportional to non-extensive Tsallis distribution when the entropic parameter $q$ is very close to unity.

For a pure self-gravitating system Sakagami and Taruya [19] have shown that, extremizing non-extensive Tsallis entropy, a stellar polytrope distribution can be obtained. This polytrope function is regarded as a finite isothermal polytrope, usually as an equilibrium state. However, the one-particle distribution function of stellar polytrope shows velocity spatial dispersion. Therefore it is no longer the equilibrium but the quasi-equilibrium state (metastable state).

A stellar core with $n, T$ and $M$ different from the $n_{*}, T_{*}$ and $M_{*}$ equilibrium values can more properly be described by a non-extensive distribution of a metaequilibrium state, rather than by the Maxwellian equilibrium distribution (that can be seen as a zero approximation).

Temperature fluctuations $\Delta T / T$ of order between $1 \%$ and $8 \%$ have been invoked to analyse neutrino oscillation parameters $[25,26]$. It is straightforward to show that fluctuations of such an order of magnitude are related to values of $\delta$ of about $10^{-4} \div 10^{-3}$. With the use of these small deformations, we do not change the macroscopic features of the core and its bulk properties; with the modified nuclear rates, we evaluate neutrino fluxes in agreement with the measured ones and, within actual uncertainties, these deformations should be taken into consideration for a more precise determination of the oscillation parameters.

This kind of fluctuation could be ruled out in an electron-nuclear plasma, after analysis of experimental results and global fits. Fluctuations of other intensive quantities can be responsible of metastable states. Fluctuating quantities can be, for instance, effective chemical potentials or functions of fluctuating energy dissipation in the flow (for the turbulence application).

Other effects that can be responsible of deviations from global thermodynamical equilibrium should be investigated, as reported below.

Li and Zhang [27] have discussed how solar core may be far from equilibrium, but rather in a quasi-stationary state, investigating the self-generated magnetic fields in the solar core [28]. In thermal nuclear fusion plasmas, where inelastic ions collisions (e.g. $p p$ chain) produce thermal energy, dissipative effects of thermal Bremsstrahlung-radiation loss-emission cause a stellar core state which differs from a global equilibrium state. Elastic collisions frequency 
of the order of $10^{17} \mathrm{~Hz}$ would destroy collective motion and coherent structure in the plasma if the solar system were isolated adiabatically. In the case of an isolated system, the Sun would be in thermodynamical equilibrium. However, the solar system is an open system and the nonequilibrium effect cancels the collision effect. Consequences are that nonequilibrium can be a source of order, the existence of random radiation fields (random electric microfields distribution and magnetic field distribution) are due to self-organization phenomena. For a discussion of the rôle of random electric microfield component see Refs. [13,29,30]; see also Jiulin Du [31] for a discussion on self-gravitation systems.

Grandpierre [32] has discussed how the core is neither a ball of ideal gas, nor a quiescent steady-state fusion reactor, but a complex self-organizing system.

Burgess et al. [33], among others, report on the importance of the resonance between g-modes and magnetic Alfvén waves in the solar radiative zone, how strongly density variations affect the solar neutrino survival probability and how oscillation parameters depend on the magnitude of solar density fluctuations.

The presence of at least one of these effects can influence the statistical distribution function and be the cause of deviations from Maxwellian distribution. We argue that these effects can be taken into account by the appropriate value of the entropic parameter $q$ [34].

\section{Conclusions}

The system we have considered in this work is the electron-nuclear plasma of the core of Sun-like stars. We can deduce that the larger is the magnitude of the quantity $\left(\mathrm{k}_{\mathrm{B}} T\right)^{3} n^{-1 / 2}$, the nearer the system is to the globally thermodynamically stable equilibrium state, because the number of particles inside the Debye-Hückel sphere increases and reaches its maximum as $\left(\mathrm{k}_{\mathrm{B}} T\right)^{3} n^{-1 / 2}$ has the maximum value. In this case, the use of the Boltzmann-Gibbs statistics is fully justified and the free energy has a minimum; also the free energy of non-extensive statistics has a minimum.

We have looked for the minimum of free energy density as stated by Sewell to find the global equilibrium states. Considering the corrections to an ideal gas due to identity of particles and to inter-nuclear interaction and the blackbody radiation emitted, by minimizing the free energy density of the electronnuclear plasma, we have obtained the following values

$$
n_{*} \simeq 2.74 \cdot 10^{-14} \mathrm{fm}^{-3}, \mathrm{k}_{\mathrm{B}} T_{*} \simeq 5 \cdot 10^{-3} \mathrm{MeV} \text { and } R_{*} \approx 0.2 R_{\odot},
$$


with a typical chemical composition $\bar{Z}=1.25$.

States with different values of $\mathrm{k}_{\mathrm{B}} T$ (lower) and $n$ (higher) are metastable states that can be featured by temperature fluctuations or density fluctuations, by quasi-particle models or by the presence of self-generated magnetic fields

or random microfields distributions. These physical effects are responsible of metastable distributions that are given by expressions that may be derived from the non-extensive thermostatistics.

Deviations from momentum Maxwellian distribution are quite small for the astrophysical systems considered in this paper and the main properties of the stars do not change at all for these deviations. Nevertheless, very slight deviations can sensibly affect the evaluation of nuclear fusion rates (as we have shown elsewhere both for nuclear astrophysics processes and for heavy ion physics and quark-gluon plasma properties) and could be useful in solving the problem of lithium abundance in the universe, among many other applications.

Supported in part by MIUR-PRIN2003.

\section{References}

[1] L. Landau and E. Lifšits, Statistical Physics, Pergamon Press, London-Paris, 1959, p. 63.

[2] G. L. Sewell, Phys. Rep. 57, 307 (1980).

[3] G. Wilk and Z. Włodarczyk, Phys. Rev. Lett. 84, 2770 (2000).

[4] C. Beck and E. G. D. Cohen, Physica A 322, 267 (2003); cond-mat/0312399.

[5] M. Coraddu, M. Lissia, G. Mezzorani and P. Quarati, in Theoretical nuclear physics in Italy, World. Sci., Singapore 2001, p. 377; nucl-th/0012002.

[6] C. Tsallis, J. Stat. Phys. 52, 479 (1998); Physica A 305 (2002), special issue, G. Kaniadakis, M. Lissia and A. Rapisarda Eds.; Non-extensive Entropy - Interdisciplinary applications, M. Gell-Mann and C. Tsallis Eds., Oxford University Press, Oxford, 2003; F. Baldovin, E. Brigatti and C. Tsallis, cond-mat/0302559.

[7] L. Landau and E. Lifšits, Statistical Physics, Pergamon Press, London-Paris, 1959, Chapt. XI p. 331.

[8] B. V. Vasiliev, Nuovo Cimento 116 B, 617 (2001).

[9] J. Bahcall, Neutrino astrophysics, Cambridge University Press, Cambridge, 1998, p. 137. 
[10] F. Ferro, P. Quarati, to be published in Phys. Rev. E.

[11] J. Bahcall, L. Brown, A. Gruzinov and R. Sawyer, Astron. Astrophys. 383, 291, (2002); (see also replies by: G. Shaviv, astro-ph/0010152 A. Lavagno and P. Quarati, astro-ph/0010055).

[12] M. Nauenberg, Phys. Rev. E 67, 036114 (2003); (see also reply by: C. Tsallis, cond-mat/0304696).

[13] A. Lavagno and P. Quarati, Phys. Lett. B 498, 47 (2001).

[14] W. M. Alberico, A. Lavagno and P. Quarati, Eur. Phys. J. C 12, 499 (2000); Nucl. Phys. A 680, 94 (2000); A. Lavagno, Phys. Lett. A 301, 13 (2002).

[15] B. V. Vasiliev and V. L. Lyuboshits, Physics-Uspekhi 37 (4), 345 (1994).

[16] D. D. Clayton, Am. J. Phys. 54 (4), 354 (1986).

[17] M. Nauenberg and V. Weisskopf, Am. J. Phys. 46 (1), 23 (1978).

[18] R. Balian and J. P. Blaizot, Am. J. Phys. 67 (12), 1189 (1999).

[19] M. Sakagami and A. Taruya, Phys. Rev. Lett. 90, 181101 (2003); M. Sakagami and A. Taruya, cond-math/0310082.

[20] J. Bahcall, M. Pinsonneault and S. Basu, Ap. J. 555, 990 (2001).

[21] V. Castellani et al., Phys. Rep. 281, 304 (1997).

[22] D. D. Clayton, Principles of stellar evolution and nucleosysthesis, Mc. GrawHill, New York, 1968.

[23] A. R. Plastino, A. Plastino and C. Tsallis, J. Phys A.: Mat. Gen. 27, 5707 (1994).

[24] F. Quarati and P. Quarati, Entropy 5, 239 (2003).

[25] H. Nunokawa, A. Rossi, V. Semikoz and J. Valle, Nucl. Phys. B 472, 495 (1996).

[26] M. Guzzo, P. C. de Hollanda and N. Reggiani, Phys. Lett. B 569, 45 (2003).

[27] L. H. Li and H. Q. Zhang, J. Phys. D: Appl. Phys. 29, 2217 (1996).

[28] L. H. Li, Phys. Lett. A 246, 436 (1998); Phys. Lett. A 2601999370

[29] G. Kaniadakis, A. Lavagno, M. Lissia and P. Quarati, Physica A 261, 359 (1998).

[30] M. Coraddu, G. Kaniadakis, A. Lavagno, M. Lissia, G. Mezzorani and P. Quarati, Braz. J. of Phys. 29 (1999) 153.

[31] Jiulin Du, to be published in Phys. Lett. A.

[32] A. Grandpierre and G. Agoston, astro-ph/0201308 A. Grandpierre, astro-ph/0201388.

[33] P.C. Burgess et al., hep-ph/0312345.

[34] C. Beck, cond-mat/0312134. 